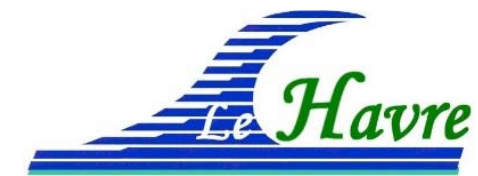

XVI İmes Journées Nationales Génie Côtier - Génie Civil

Le Havre, 2020

DOI:10.5150/jngcgc.2020.074 @ Editions Paralia CFL

disponible en ligne - http://www.paralia.fr - available online

\title{
Transportabilité des sédiments: un essai de pelletabilité
}

\section{Daniel LEVACHER ${ }^{1}$, Gilles FRIBOULET ${ }^{2}$, Coryse COUDRAY ${ }^{2}$, Sylvain HAQUIN ${ }^{3}$}

1. Normandie Université, Unicaen, CNRS UMR 6143 M2C, 14000, Caen, France. daniel.levacher@unicaen.fr

2. EDF - R\&D, TREE E34 groupe Territoires et Economie Circulaire, Avenue des Renardières, 77818 Moret-sur-Loing cedex, France.

gilles.friboulet@edf.fr ; coryse.coudray@edf.fr

3. Ingénieur-conseil, 44200 Nantes, France.

sylvain.haquin@ec-nantes.fr

\section{Résumé :}

La teneur en eau élevée des sédiments de dragage constitue un réel frein à toute valorisation matériau de ceux-ci. Elle l'est aussi pour le transport des sédiments dragués depuis des lieux difficilement accessibles comme les barrages, les lacs, les fleuves, les bassins portuaires... Ce transport nécessite de pouvoir manipuler les sédiments pour les charger : ils doivent être pelletables. Mais la notion de pelletabilité des sédiments reste à définir à partir de critères basés sur des paramètres intrinsèques des sédiments. Certains paramètres apparaissent en effet appropriés pour définir un ou plusieurs critères d'aptitude à la pelletabilité. Une fois retenus, ces paramètres doivent être déterminés par leur mesure directe ou déduits d'autres mesures. Cet ensemble de mesures implique la définition d'un essai spécifique dédié à la pelletabilité des sédiments. C'est l'objet de cette communication. Après avoir présenté les paramètres physiques et mécaniques propres à la pelletabilité des sédiments, un concept pour un essai de pelletabilité est proposé. Un premier prototype d'essai en version manuelle est décrit tout comme la procédure liée à son utilisation. Ce même prototype avec la même conception pourra être équipé pour une version automatisée sans aucune modification majeure. Cet équipement d'essai spécifique à la pelletabilité des sédiments pourra être adapté pour d'autres applications.

Mots-clés :

Sédiments à teneur en eau élevée, Dragage, Déshydratation, Déshydratation mécanisée, Essai de pelletabilité, Séchage, Stabilisation, Travaux maritimes, Transportabilité, Valorisation matière. 


\section{Thème 6 - Gestion durable des zones littorales et estuariennes}

\section{Introduction}

L'abattement de la teneur élevée des sédiments à l'issue de leur dragage est un préalable nécessaire pour assurer leur transport et favoriser les processus de stabilisation (figure 1). Excepté si l'on peut recourir à un réemploi direct de ces sédiments ou à une stabilisation sur place, un processus de déshydratation doit être mis en œuvre en raison de la difficulté des opérations de transport, de terrassement et de stabilisation ultérieure. Cette déshydratation, qu'elle soit naturelle ou mécanisée, consiste la plupart du temps à éliminer l'eau en vue d'en abaisser la teneur dans les sédiments. Il est aussi possible d'assurer non pas une déshydratation par élimination directe de l'eau, mais par un transfert de l'eau de la matrice sédimentaire vers des matières solides très absorbantes ajoutées aux sédiments. Ces matières solides une fois imbibées permettent, selon leur quantité ajoutée, dans un premier temps de manipuler les sédiments puis de pouvoir les transporter. Et de plus, si le choix de ces matières solides est judicieux, elles permettent par la suite d'améliorer les performances mécaniques de ces sédiments stabilisés en vue de réaliser des aménagements ou ouvrages de construction (voies piétonnes, routières, remblais, ouvrages en terre...). Ces matériaux absorbants peuvent être des déchets fibreux naturels.

Le réemploi direct de sédiments à haute teneur en eau est courant en travaux maritimes. A très haute teneur en eau, les sédiments sont liquides et sont aptes à être pompés. Cet état permet le refoulement de sédiments à terre dans des zones prévues à cet effet lors de dragages portuaires. Mais pour un réemploi direct avec valeur ajoutée, ces sédiments sont additionnés de liants et d'agents de viscosité afin de réaliser une stabilisation des sédiments différée dans le temps de manière à assurer leur transport par pompage. Nous pouvons citer les applications récentes de confection de noyaux de digues, de plateformes de remblais et de réalisations de systèmes anti-affouillement à la base de mono-pieux d'éoliennes en mer (WANG, 2019). La stabilisation sur site de sédiments a aussi fait l'objet de nombreux travaux et d'études, notamment pour des sites de sédiments contaminés. Différents procédés de stabilisation sur site ont été développés, nous pouvons citer l'utilisation de l'hydro-fraise et les techniques ALLU (TREVES, 2010). Pour supprimer le frein à la valorisation matière dû à la teneur en eau élevée des sédiments, des techniques de déshydratation mécanisée en continu par élimination de l'eau adaptées à des volumes conséquents de sédiments, ont été développées cette décennie. Nous rappelons ici différents systèmes disponibles sur le marché : Némeau, Volute, Ec'Eau Wave et Doris (BOULLOSA ALLARIZ, 2018 ; HYTRASED, 2019). La déshydratation par apports solides fibreux a fait l'objet de récentes applications notamment en utilisant des fibres de bambou (KAGO et al., 2019). Ce transfert d'eau aux fibres plus ou moins enchevêtrées permet d'assurer une meilleure pelletabilité des sédiments qui pourront être stabilisés par la suite avec des liants. Dans cette technique, l'eau n'est pas totalement éliminée mais la réduction opérée permet d'envisager une stabilisation comme méthode d'amélioration et cette technique n'altère en rien les propriétés physiques du sédiment. 


\section{XVİ̀mes Journées Nationales Génie Côtier - Génie Civil \\ Le Havre 2020}

Mais en pratique, les machines de déshydratation et engins de terrassement ayant difficilement accès aux sites de dragage, les sédiments doivent être pelletés pour être transportés et rassemblés sur un site de stockage et de valorisation. Tout en supposant que les techniques de déshydratation soient maîtrisées et adaptées aux sédiments, une question se pose alors: à quelle teneur en eau peut-on espérer pelleter et/ou charger des sédiments?
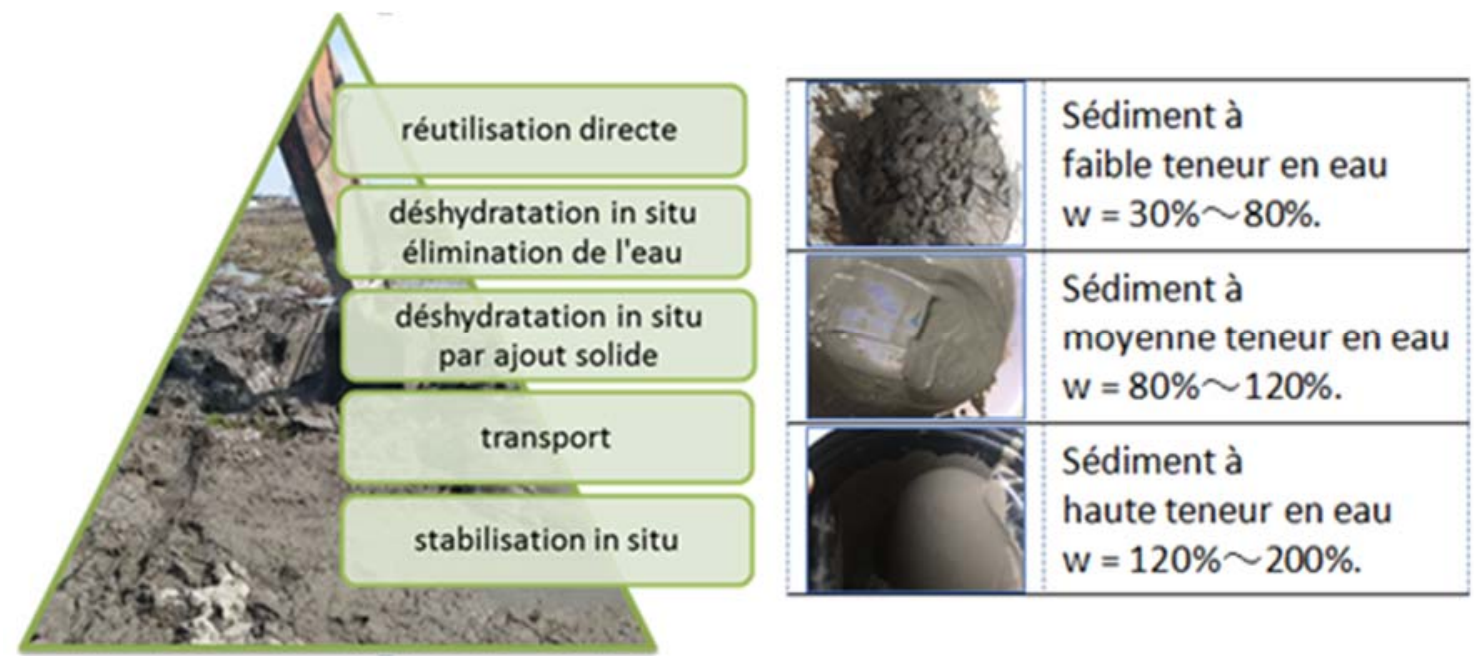

Figure 1. Prétraitements potentiels de sédiments à teneur en eau élevée, d'après WANG, 2019.

\section{Pelletabilité des sédiments}

\subsection{Paramètres gouvernant le phénomène}

La littérature fournit peu de recommandations relatives à cette notion de pelletabilité des sédiments. De même aucune classification associée à la teneur en eau (w) n'est établie à propos des sols ou sédiments. Une proposition a été donnée par WANG (2019) qui délimite trois catégories de teneurs en eau (figure 1).

Quant à la pelletabilité des sédiments une suggestion a été émise par MANCIOPPI et al., (2015) situant les performances d'un outil de déshydratation utilisé pour des sédiments (figure 2). 


\section{Thème 6 - Gestion durable des zones littorales et estuariennes}

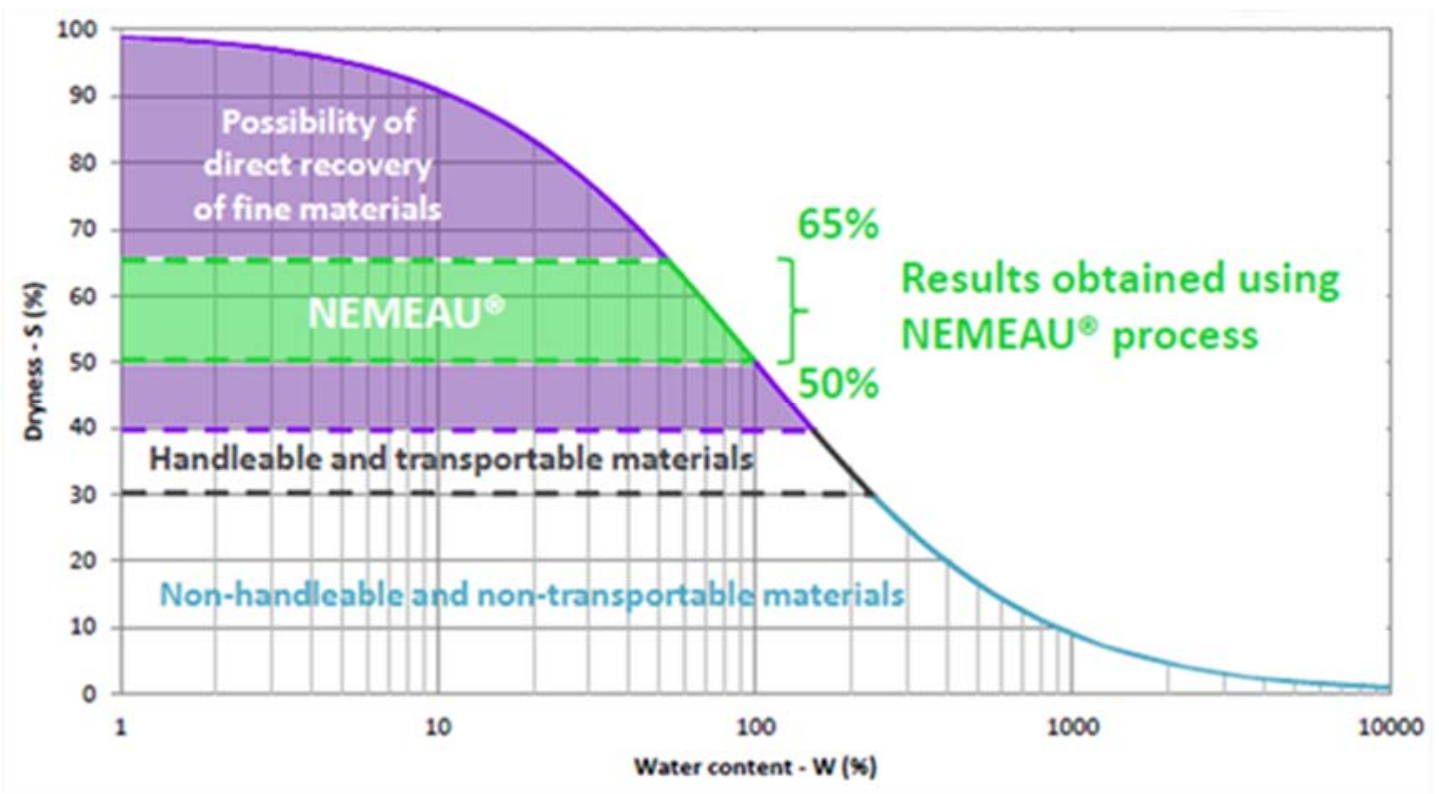

Figure 2. Zones de pelletabilité, de déshydratation et de stabilisation des sédiments, (MANCIOPPI et al, 2013).

Les paramètres qui gouvernent les phénomènes de pelletabilité sont d'une part les caractéristiques d'état du sédiment pendant son séchage naturel (teneur en eau w, indice des vides e, degré de saturation $\mathrm{S}_{\mathrm{r}}$ ) et de consistance (limites d'Atterberg WL et WP, indices de plasticité $I_{P}$, de liquidité $I_{L}$ et de consistance $I_{C}$ ). Mais d'autre part des propriétés mécaniques interviennent comme la cohésion non drainée du sédiment $\left(\mathrm{S}_{\mathrm{u}}\right)$ et sa limite d'adhérence $\left(\mathrm{L}_{\mathrm{A}}\right)$. Cette dernière peut se définir comme étant la teneur en humidité à laquelle le sol adhère à la surface d'un métal. La cohésion peut être reliée à la consistance des sédiments par la mesure de l'affaissement (A). Ces paramètres ont été déjà utilisés dans des études de séchage de sédiments (SERRATRICE, 2015; BOULLOSA ALLARIZ et al., 2019; HASSAN et al.,2020), de transport de sols (PRABHAKAR \& SATYESWARARAO, 2012) et de pompabilité de sédiments en cours de stabilisation (WANG, 2019). 


\section{XVIèmes Journées Nationales Génie Côtier - Génie Civil \\ Le Havre 2020}

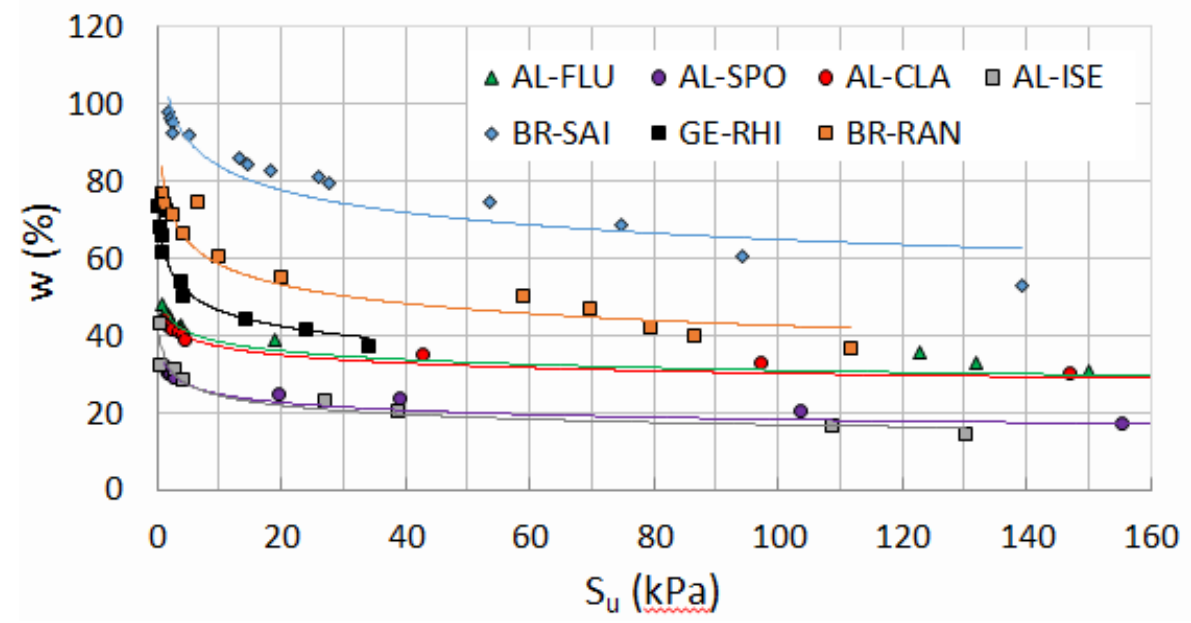

Figure 3. Relation teneur en eau-cohésion non drainée $S_{u}$ pour des sédiments,

(BOULLOSA ALLARIZ et al., 2019).

\subsection{Conception d'un essai de pelletabilité}

La mise au point d'un essai spécifique de pelletabilité a été basée sur la mesure d'un ensemble de paramètres régissant la pelletabilité des sédiments, à savoir la cohésion non drainée $\left(S_{u}\right)$ et/ou l'affaissement $(A)$, la teneur en eau $(w)$ et la limite d'adhérence $\left(L_{A}\right)$. Ce dernier paramètre est à relier avec la rugosité $\mu$ du support métallique et de son inclinaison $\beta$. La procédure est illustrée à la figure 4 . Tout d'abord le paramètre initial qui reste constant pendant le déroulement de l'essai est la teneur en eau w du sédiment. S'ensuit une succession de mesures comme l'affaissement A du sédiment et la mesure de la cohésion non drainée (utilisation d'un scissomètre de laboratoire ou éventuellement de l'essai au cône à chute libre, AFNOR (2017), BS (2018), HANSBO (1957); SHIMOBE \& SPAGNOLI (2020), voir figure 4-1. L'étude de l'adhérence et du glissement du sédiment sur une plaque métallique de rugosité connue se fait par la mesure de l'angle $\beta$ durant la mise en rotation de celle-ci, (figure 4-2). 


\section{Thème 6 - Gestion durable des zones littorales et estuariennes}
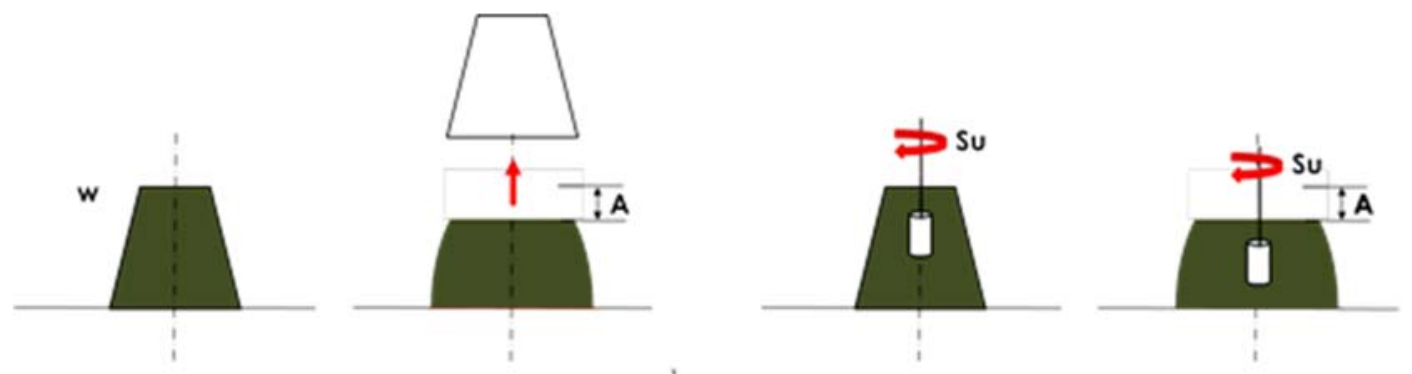

Figure 4-1. Concept de l'essai de pelletabilité.
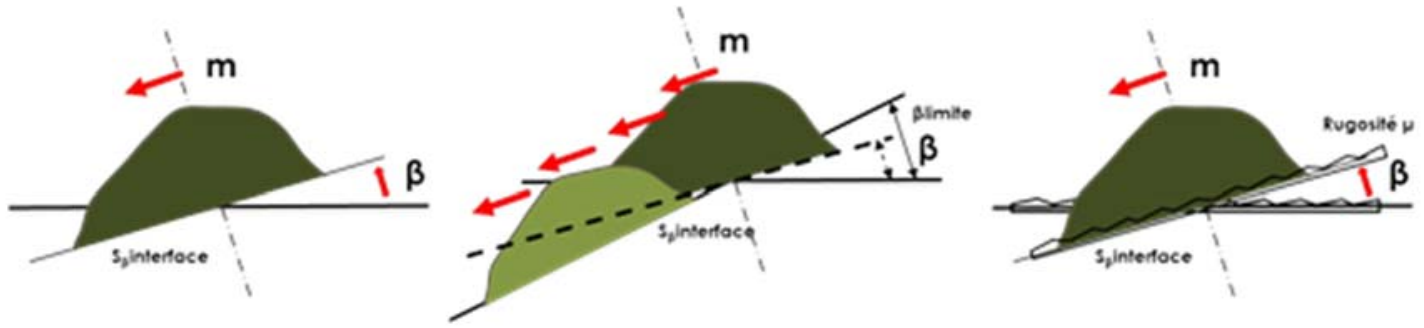

Figure 4-2. Concept de l'essai de pelletabilité.

A partir du concept et de la procédure ainsi définis, un prototype d'essai a été conçu et une version manuelle proposée. Ce prototype est montré à la figure 5, avec ses différentes pièces le constituant. On remarquera que la plaque métallique est interchangeable pour modifier la rugosité et qu'un suivi de la cinématique de la masse des sédiments est prévu avec une caméra rapide pour une exploitation des images.

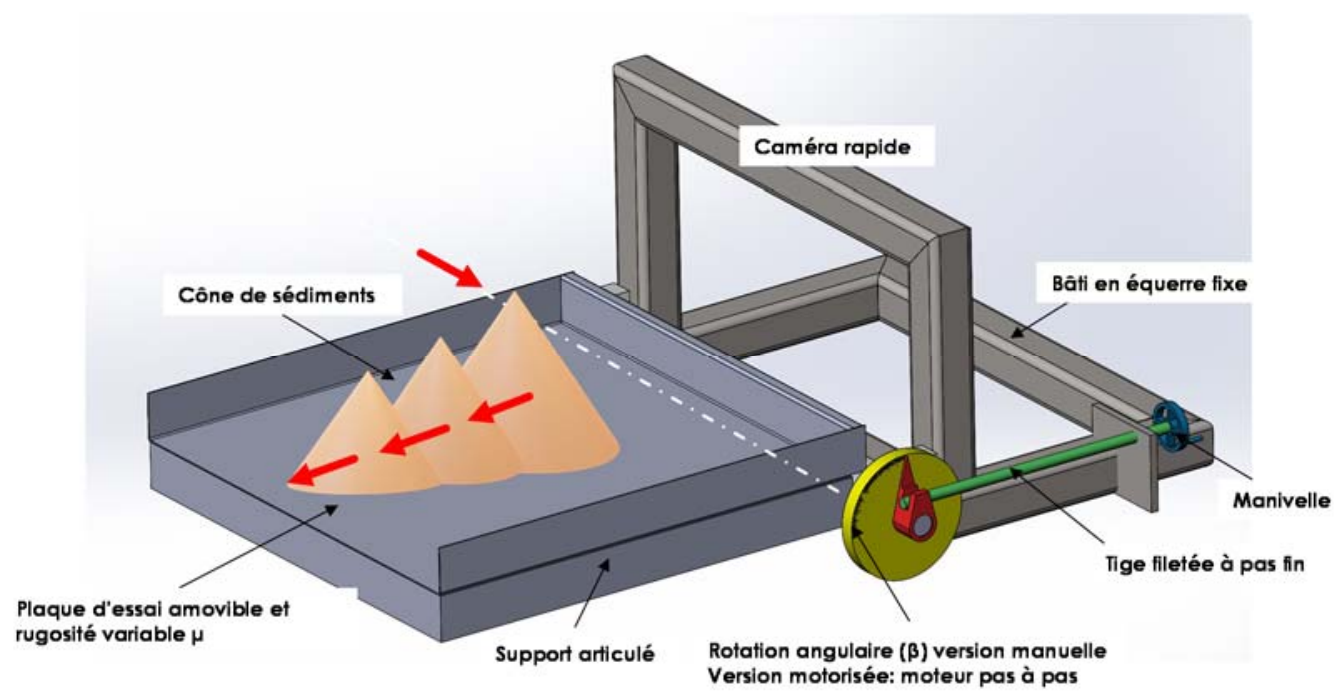

Figure 5. Prototype de la version manuelle de l'essai de pelletabilité, HOST ${ }^{\circledR}$ test, LEVACHER, 2020. 


\section{XVI'̀mes Journées Nationales Génie Côtier - Génie Civil \\ Le Havre 2020}

\section{Conclusions}

Cet essai de pelletabilité de sédiments est tout d'abord envisagé pour recueillir un ensemble de données qui permettront de comprendre les mécanismes liés à la pelletabilité mais plus précisément de définir des critères de pelletabilité ou d'aptitude au transport des sédiments. Ce type d'essai ou d'appareillage peut être utile à d'autres études avec des matériaux équivalents (sols, poudres,..) comme il peut être adapté sur un canal à houle pour étudier la mise à l'eau de gabions ou le déversement de matériaux agglomérés pour réaliser un remplissage ou une couche de protection sur des fonds marins. Cette version manuelle de prototype sera automatisée par la suite.

\section{Références bibliographiques}

AFNOR (2017). Reconnaissance et essais géotechniques - Essais de laboratoire sur les sols - Partie 6: Essai de pénétration de cône, ISO 17892-6:2017 février 2017.

BOULLOSA ALLARIZ B. (2018). Déshydratation naturelle et mécanisée de sédiments - Étude des processus mis en jeu et applications, Thèse de doctorat Université Caen Normandie, 427 p. HAL Id : tel-02292209

BOULLOSA ALLARIZ B., LEVACHER D., MÜLLER M. (2019). Étude du séchage naturel des sédiments du port de Dunkerque en vue de valorisations ultérieures. Déchets sciences et techniques, $\mathrm{N}^{\circ} 80$. https://doi.org/10.4267/dechets-sciences-techniques.4099

BS (2018). Geotechnical investigation and testing. Laboratory testing of soil. Determination of liquid and plastic limits. EN ISO 17892-12:2018.

HANSBO S. (1957). A new approach to the determination of the shear strength of clay by the fall cone test. R. Swedish Geotechnical Institute Proceedings, 14, pp 5-47.

HASSAN A., LEVACHER D., MEZAZIGH S. (2020). Controlled dewatering, transportability and valorization of dredged sediments, Rapport EDF-M2C, Ref. $\mathrm{N}^{\circ} \mathrm{M} 2 \mathrm{C}-\mathrm{HYTRASED}-\mathrm{EDF}-03,99 \mathrm{p}$.

HYTRASED (2019). Pelletabillité, transport et déshydratation de sédiments, Projet collaboratif, Université de Caen Normandie, Laboratoire M2C, 15 p.

KOGA C., SATO K., FUJIKAWA T. (2019). Improvement effect of high water content dredged clay using bamboo chip and cement. $16^{\text {th }}$ Asian Regional Conference on Soils Mechanics and Geotechnical Engineering, Taipeh, Taiwan, 4 p.

LEVACHER D. (2020). Handling of sediment test design, HOST ${ }^{\circledR}$ test, version manuelle, Projet collaboratif, Université de Caen Normandie, Laboratoire M2C, 12 p.

MANCIOPPI L., DHERVILLY P., LEVACHER D., (2013) Breaking technology for dewatering and valorization of sediment in France- Process Nemeau. $20^{\text {th }}$ World Dredging Congress and Exhibition 2013, WODCON XX, The Art of Dredging, Brussels, Belgium, Vol. 1/2, pp 584-589.

PRABHAKAR Y. S., SATYESWARARAO B. (2012). Earth work handling problems due to stickiness of soils - sticky limit - evaluation \& measurement methods. TRACE- 


\section{Thème 6 - Gestion durable des zones littorales et estuariennes}

2012 conference, National Conference Proceedings, Amity School of Engineering \& Technology, Amity University, UP, Noida, India, Vol. 1, pp 8-19.

SERRATRICE J-F. (2015). Une représentation des courbes de retrait des sols argileux. SEC 2015 International Symposium, IFSTTAR, Marne-la-Vallée, pp 179-186.

SHIMOBE S., SPAGNOLI G. (2020). Fall cone tests considering water content, cone penetration index, and plasticity angle of fine-grained soils. Journal of Rock Mechanics and Geotechnical Engineering. https://doi.org/10.1016/j.jrmge.2020.02.005

TREVES S. (2010). Traitement et valorisation des sédiments marins. XI ${ }^{\text {ème }}$ Journées Nationales Génie Côtier - Génie Civil, Les Sables d'Olonne, pp 909-912. https://doi.org/10.5150/jngcgc.2010.098-T

WANG X. (2019). Méthodologies de valorisation de sédiments mises en œuvre dans des ouvrages géotechniques en Chine. Thèse de doctorat, Université de Caen Normandie, 202 p. https://tel.archives-ouvertes.fr/tel-02520015v1 